\title{
Data-Driven Model Based Charging Profile Prediction for Energy Storage Systems
}

\author{
Nandha Kumar Kandasamy ${ }^{1,{ }^{*}}$ and Rudy Tjandra ${ }^{2}$ \\ ${ }^{1}$ iTrust, Singapore University of Technology and Design, Singapore \\ ${ }^{2}$ Maritime Institute@ Nanyang Technological University, Singapore
}

\begin{abstract}
Energy storage systems (ESS) are penetrating into various sections of power system through different applications. ESS can be used either as a buffer for intermittent renewable energy sources or as a stand-alone distributed storage for load shifting. ESS use different types of storage devices such as lead-acid batteries, lithium ion batteries, flow batteries, and super-capacitors. Hybrid ESS consisting of few types of storage devices are also common in practice. Determining the load demand of such ESSs at various instances (charging profile) accurately is indispensable in most of the cases. Capacity loss is common phenomenon that occurs in all types of storage devices because of ageing. Capacity loss has to be accounted while determining the charging profile of storage devices for better accuracy. Data-driven modeling is an attractive approach for determining the load demand of ESS due to the availability of valuable data from smart grid technologies. In this paper, the application of different types of data-driven models to predict the current charging profile of the ESS based on previous charging profiles is examined. The proposed method can leverage on the existing data from smart grid and is a black box modeling approach.
\end{abstract}

Keywords-energy storage systems; charging profile; capacity loss; data-driven modeling

\section{INTRODUCTION}

The prolific growth in the penetration of energy storage systems (ESS) into distribution network in the recent past can be attributed to climate change policies of many countries including Singapore $[1,2]$. The Singaporean government has released tenders for installing more solar PV systems and it is estimated that solar PVs will be installed in many Housing Development Board (HDB) blocks and Public Sector buildings by 2017 [3]. However, large-scale integration of such intermittent renewable energy sources will affect the reliability and stability of the distribution network [4-9]. Utilizing ESS as a buffer for intermittent renewable energy sources will reduce the variability in power output of such sources and hence the impact on reliability and stability [1012]. Using ESS provide a multitude of benefits to utilities including peak load management, load shifting applications (peak shaving and valley filling), voltage regulation and power quality improvement [13]. The ESS are to be operated both as source and load while executing functions such as load shifting. Apart from these applications, most modern day mobile devices are equipped with ESS. Determining load demand/charging profile of appliances having large storage devices such as electric vehicles (EVs) is also critical [14, 15]. The charging profile of a storage device varies based on various parameters such as initial State Of Charge (SOC), final desired SOC, storage device type and charging/discharging cycles (corresponds to capacity loss) completed. Accurately determining the charging profiles of such storage devices connected to a distribution network will enable various energy management applications such as maximum demand prediction, load scheduling and demand response management.

\section{RELATED WORK}

The charging of any storage devices is a nonlinear process and changes regularly due to the change in chemical composition of the device $[16,17]$. Capacity loss due to ageing process is an important factor that affects the charging profiles of any storage device $[16,17]$. The authors of [1620] have studied the ageing process of lithium ion and vanadium flow batteries. They have proposed ageing models that were based on accelerated ageing. The models can be utilized for determining the capacity fade and could be combined with the charging power model proposed in [15] for determining the theoretical charging power of the whole charging process of any ESS. However, the constants derived based accelerated ageing process may not match the practical values due to differences in various operating parameters such as temperature, usage pattern, location of the device etc. This disadvantage could be eliminated by updating the parameters online after certain number of cycles. However, storage devices employed in smart grids have the inherent advantage that the power consumption data is continuously monitored. In such cases data-driven modeling could be used to determine the charging profiles of energy storage devices [14, 15] (a black box approach). In this paper, data-driven models based on different methods are used determine the charging profiles of ESS used for different applications. The existing models from the accelerated ageing experiments are used for determining the charging profiles and the values of the ESS parameters are varied to portray the variations in actual scenarios. The accuracy of the model to predict the charging profiles is subsequently evaluated. The models are also 
applied to predict the charging profiles of an $1100 \mathrm{mAh}, 3.6 \mathrm{~V}$ lithium iron phosphate battery, charging profiles were collected over complete life cycle ( $\sim 3500$ cycles) for nonaccelerated ageing study. It is to be noted that the contribution of the paper pertains to the approaches used for determining charging profile/load demand of energy storage devices rather than the data-driven models. Leveraging on the existing data is an important aspect that would add value to the smart grid infrastructure. To summarize the contributions of the paper, the paper studies the application and comparison of different types of data-driven models that can be used for ESS and evaluates the approach of using generalized data-driven models for ESS used in different applications. A black box modeling approach is used for the application similar to the studies in authors' previous work [21].

\section{DATA REQUiRED For MODELING}

\section{A. Simulated Data:}

The capacity fade model for lithium iron phosphate batteries proposed in [17] and charging power model proposed in [15] are used to obtain the charging profiles (for the whole life cycle) ESS in following applications

\section{a) ESS in a Personal EV:}

An EV with a $24 \mathrm{kWh}$ battery capacity (representing Nissan Leaf) is considered for the study. The mean distance travelled in a day is taken as $55 \mathrm{~km}$ [22-24] and standard deviation of $10 \mathrm{~km}$ is considered. Based on a stochastic model and average energy consumption $/ \mathrm{km}$, the energy required per day and initial SOC on each day is computed. This application also represents other types of ESS with CC charging/CV charging that is used for devices with mobility. E.g., Laptop PCs, tablets, mobile phones, emergency lamps, emergency power supply (power banks for mobile applications) etc. ESS without any mobility but needing fixed charging operations such as emergency power supply ESS for residential/commercial buildings, ESS used for peak shaving/peak shifting applications etc. can also be categorized under such charging pattern.

\section{b) ESS used for a typical solar photovoltaic application:}

$(P V)$

An ESS with $24 \mathrm{kWh}$ battery energy capacity and $48 \mathrm{~kW}$ power capacity for typical roof top PV of $70 \mathrm{~kW}_{\text {peak }}$ is considered for the study (sizing of the ESS is not within the scope). The power/energy consumption and corresponding SOCs are obtained using solar irradiance data for a period of 1 year (2010). The charging profile is determined for the application wherein the PV uses the ESS to maintain the average power supplied throughout the day (9am-5:30pm). The charging/discharging power is obtained from the difference between the actual output of solar PV and the average output. The difference from the previous application is that the charging power is not a constant value and it varies based on the PV output. This application also represents ESS used for other type renewable energy sources and ESS used for frequency regulation etc.

\section{B. Experimental Data:}

The charging profiles of $1100 \mathrm{mAh}, 3.6 \mathrm{~V}$, lithium iron phosphate battery are collected for complete life cycle ( $\sim 3500$ cycles). The battery is cycled using a constant discharging at $1500 \mathrm{~mA}$ and charging with $1500 \mathrm{~mA}$ in $\mathrm{CC}$ and decreasing current to maintain the voltage in $\mathrm{CV}$ mode. In this case, the cycle depth is fixed and the SOC is calculated using coulomb counting. However, the data serves as an excellent base for understanding the capability of the proposed method to predict the charging profiles with minimal input parameters in practical applications. Furthermore, the data is collected for normal operation without any accelerated ageing and hence demonstrates the flexibility of the proposed method.

\section{DATA-DRIVEN MODELS}

Upspring of smart grid facilitated the availability of huge data on various power-consuming devices. The available data may be utilized for various purposes and prediction of load demand is an important application for the available data. Data-driven modeling has always been an attractive method due to one of its critical feature; namely ability to update model parameters to capture the changes due to ageing. Furthermore, scaling up or down the system is effortless in most of the cases. Data-driven modeling has been extensively utilized [25-27] for data-driven simulations and data-driven controller design. In this paper, different data-driven models that are suitable for predicting the charging profiles of energy storage systems (based on previous charging profiles) are examined. Since data-driven models have the ability to learn, it can easily adapt to changes in the charging profiles of energy storage system. Energy Storage Models based on Artificial Neural Network (ANN) with feed-forward network and Ensemble Learning (boosting) are adopted from authors' previous work [21]. Energy Storage Model based on Quadratic Polynomial Curve Fitting is presented below.

The charging profile of the ESS can be represented using quadratic polynomial equations. Quadratic polynomial curve fitting can be used as a simple solution, because a simple empirical model for characterizing the data is sufficient. Linear polynomial equations could be used for ESS without $\mathrm{CV}$ modes. However, for a generalized model that could fit all types of ESS, a Quadratic Polynomial model is essential. Polynomial models for curves are given by,

$$
y=\sum_{i=1}^{n+1} P_{i} x^{n+1-i}
$$


where, the order of the polynomial is given by $n+1$ and the degree of the polynomial is given by $n$. ' $y$ ' represents the charging power and ' $x$ ' represents the SOC of the ESS. Piecewise polynomials based on non-parametric fitting can also be used for maximizing the flexibility of the fit. Reasonable flexibility for simple data, linearity, and simple fitting process are the advantages. Instability for high-degree fits and wild divergence outside certain data-range are the disadvantages. The inputs required are obtained from previous charging profiles. However, in the proposed application, extrapolation outside the data set is not required and hence quadratic polynomial curve fitting can be applied without any limitations.

\section{Simulation RESUlts}

\section{1) Case Study 1-Energy Storage Systems in a Personal EV:}

Based on the scenario explained for application ' $A$ ' in Section III, the simulated charging profiles of the EV over its life time for ' $\emptyset$ ' $=2.5$ [21] is illustrated in Fig. 4(a) (until the capacity reaches $80 \%$ of the original value for the conditions explained in Section III (A)). The observation shows that the charging time in $\mathrm{CV}$ region decreases with an increase in the number of cycles and vice versa in $\mathrm{CC}$ region. It is an expected phenomenon as a consequence of capacity fade and the use of same charger for charging the EV. The phenomenon clearly demonstrates the capacity fading effect altering the charging profile of EVs considerably. Fig. 4(b) provides further proof on the impact due to ageing. It can be observed that although the energy required (distribution) remains almost constant, the initial SOC decreases consistently with the increase in number of cycles (the charging SOC window gets wider). Furthermore, the charging profile of the same EV/ESS changes significantly for same number of charging cycles with different values of ' $\emptyset$ ' [21] and can be inferred from Fig. 5. With increase in ageing factor (decrease in ' $\varnothing$ ') the charging time in CC region increases and vice versa in CV region. Changes in ' $\emptyset$ ' represent the changes in different driving cycles as well as difference in driving behavior. Hence, capturing the changes due to ageing factors is critical for accurately determining the charging profiles.

The prediction of charging profile is carried out for the $51^{\text {st }}$ and $1501^{\text {th }}$ charging cycle of the EV with ' $\varnothing$ ' $=2.5$ using all the ESS models. The results are presented in Fig. 6(a), (b) and (c). Notably the number of previous charging profiles used has a minimal impact (ranging from 10-50), and hence ignored. The number of previous charging profiles is fixed at '10'. From Fig. 6(a), it is evident that ANN generates a better prediction for the complete charging period, whereas curve fitting results in an inferior prediction performance. The performance of ensemble model is similar to ANN in the constant current region, however, the error increases in the constant voltage region. In case of ensemble model, the performance is similar to $\mathrm{ANN}$ in $\mathrm{CC}$ region however, the

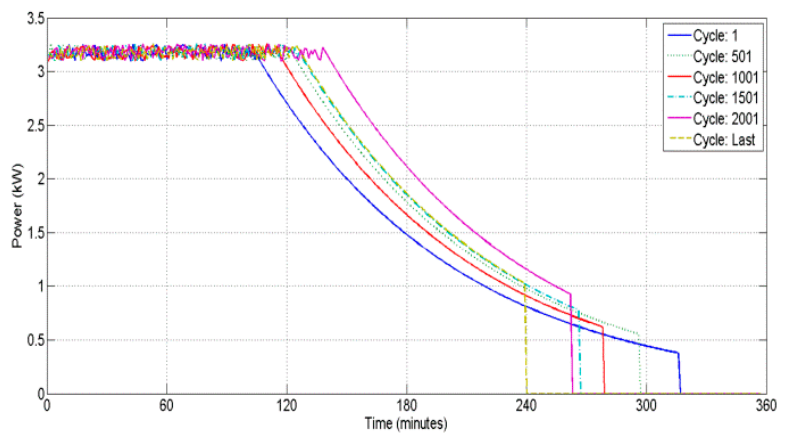

Fig. 4(a). Charging profiles of the EV over its life-time for ' $\emptyset$ ' $=2.5$

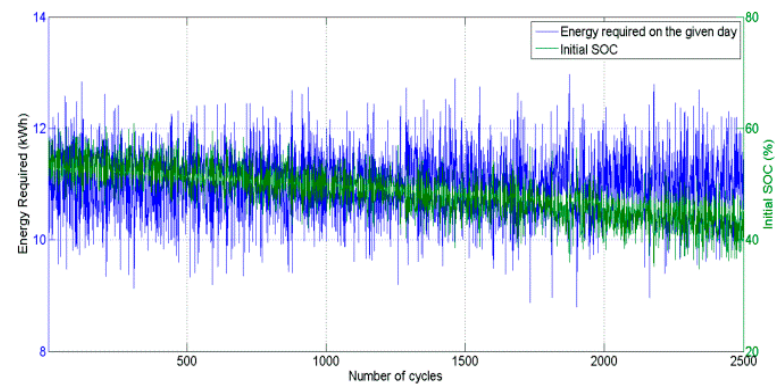

Fig. 4(b). Initial SOC and energy required of the EV over its life-time for ' $\varnothing$ ' $=2.5$

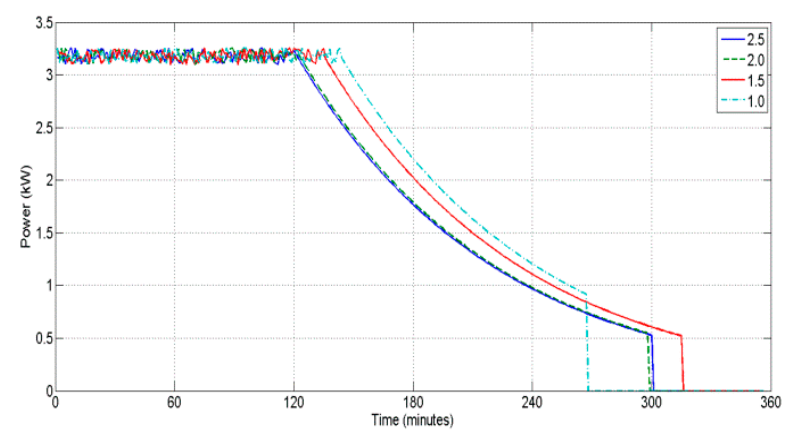

Fig. 5. Charging profile of the same EV/ESS for $251^{\text {st }}$ cycle with different values of ' $\emptyset$ ' $=2.5,2,1.5$, and 1.0

error $^{1}$ increases in the $\mathrm{CV}$ region. ANN provides a better performance in the $\mathrm{CV}$ region.

The trend remains identical for the $1501^{\text {th }}$ cycle and can be deduced from Fig. 6 (b). Hence, the number of charging cycles the ESS has undergone does not affect the prediction accuracy. In Fig. 6(c), the ability of the ANN (other models are not presented owing to the better performance of ANN) to predict the charging profiles under different ageing factors is demonstrated. It can be observed that irrespective of the value of ' $\varnothing$ ' (2.5 and 1.0 [21]) the ANN accurately predicts the charging power. It is to be noted that the 'Power Available' from the distribution system is set to maximum value and does not have any significance in applications such as EV battery charging. This is attributed to the lack of control on the power supplied to the ESS is in such applications and the charging will be completely interrupted as a part of any scheduling algorithms.

\footnotetext{
${ }^{1}$ Error analysis is carried out only for the case where experimental profiles are used.
} 


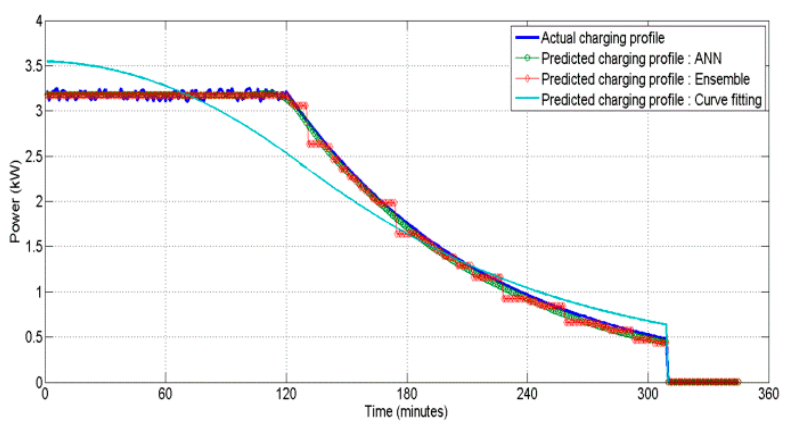

Fig. 6(a). Predicted charging profile for $51^{\text {st }}$ cycle with ' $\varnothing$ ' $=2.5$.

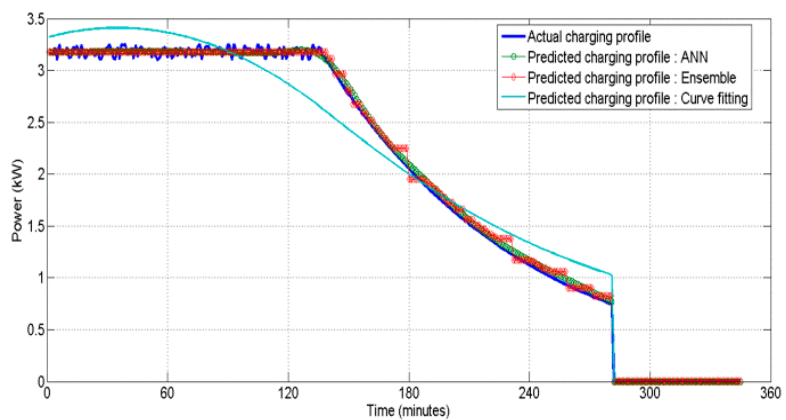

Fig. $6(\mathrm{~b})$. Predicted charging profile for $1501^{\text {th }}$ cycle with ' $\emptyset$ ' $=2.5$.

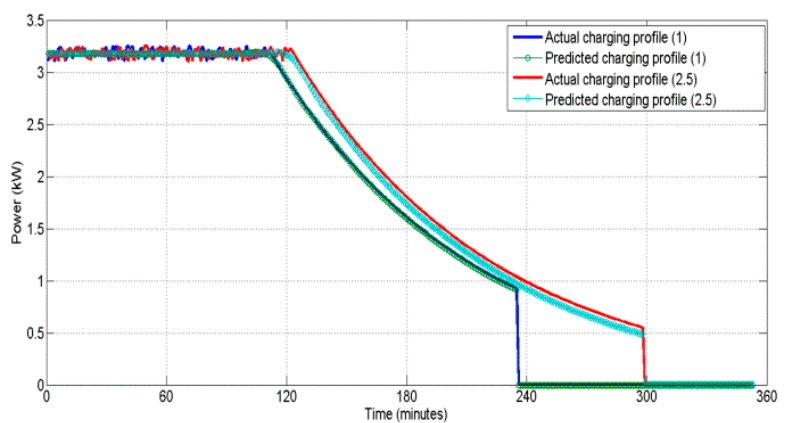

Fig. 6(c). Predicted charging profile of the same EV/ESS for $251^{\text {st }}$ cycle with different values of ' $\varnothing$ ' $=2.5$ and ' 1 ' using ANN based model

2) Case Study 2- Energy Storage System used for a typical solar photovoltaic (PV) application:

Fig. 7 illustrates the difference between charging/discharging power available due to the intermittency of solar PV and the actual charging/discharging profile of the ESS due to the limitations imposed by equation (3). It can be clearly observed that even when higher power is available for charging/discharging the actual charging/discharging charging power depends on the SOC of the ESS. The charging power reduces when SOC is greater than $80 \%$ and discontinues when SOC is $100 \%$. When the SOC reaches $20 \%$, no discharging is permitted. This clearly demonstrates the need for predicting the actual charging/discharging profile of ESS based on their SOC to activate other demand response management to balance the mismatch.

Based on the scenario explained for application ' $\mathrm{B}$ ' in Section III, the charging/discharging profiles are simulated for ' $\varnothing$ ' $=2.5$ [21]. The profiles are used for training the datadriven models that were explained in Section IV. The predicted charging/discharging profiles using different models are shown along with the actual profile in Fig. 8. 'Charging/discharging' profiles of the 10 previous days are used for training the models. The inference from Fig. 8 is that the ANN performs better than other two methods and generates a better prediction (similar to application 'A'). However, there is a fixed error at the extreme end of the SOCs i.e., $100 \%$ SOC and $20 \%$ SOC. The error could be eliminated with a simple saturation block. It was observed that increasing the number of charging profiles used for training the models did not have impact on this fixed error or did not result in rectification of the resulting error.

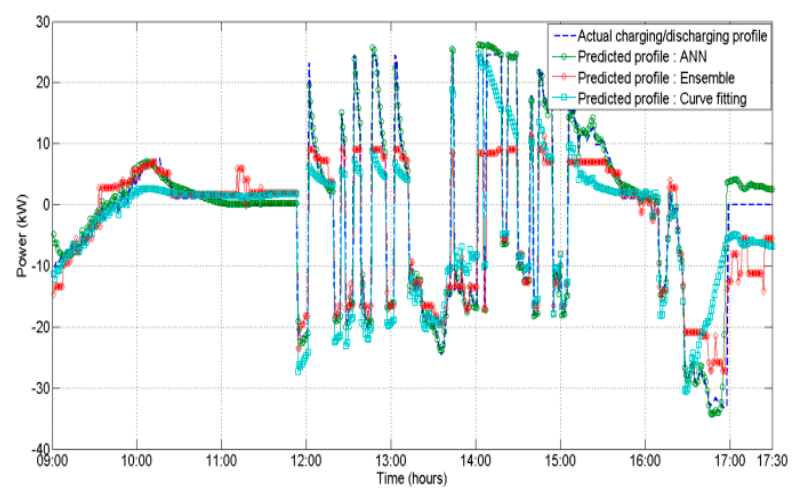

Fig. 8. Charging/discharging profile of the ESS on $131^{\text {st }}$ day of the year with ' $\varnothing$ ' $=2.5$

intermittency of solar PV and charging/discharging profile of the ESS on $130^{\text {th }}$ day of the year with ' $\varnothing$ ' $=2.5$

\section{RESUltS With EXPERIMENTAL DATA}

The charging profile of $1100 \mathrm{mAh}, 3.6 \mathrm{~V}$, lithium iron phosphate battery after 50 cycles, 1000 cycles and 3500 cycles is shown in Fig. 9. The capacity decade i.e., reduction charging time and energy consumed over the life span can be observed similar to the simulated profiles in Fig.4. 


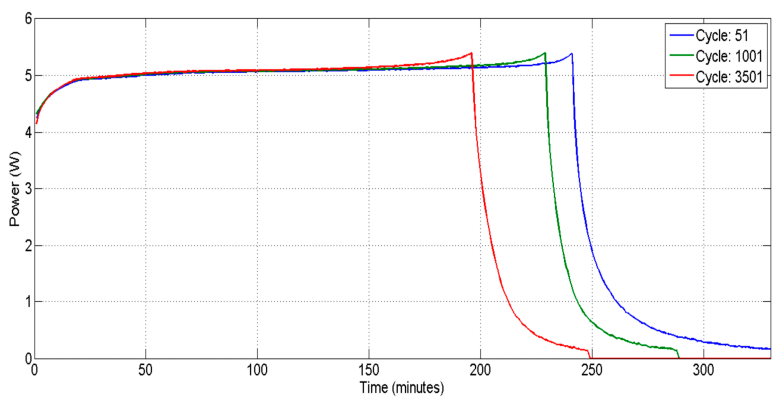

Fig. 9 Charging profiles of the battery over its life-time.

Fig. 10 (a) shows the charging profile $\left(51^{\text {st }}\right)$ predicted with models described in Section IV. As in Section V, 10 previous charging profiles are used for training. It is clearly evident from the Fig. 10(a), that ANN provides a better prediction in comparison to other methods. In Fig. 10(b) the actual and charging profiles predicted using ANN for $51^{\text {st }}$ cycle, $1001^{\text {st }}$ cycle, $3501^{\text {st }}$ cycle are shown. Similar to Section $\mathrm{V}$, the performance of the model is not affect due to ageing of the battery. A root square error (RSE) plot for comparing the accuracy of the three models at $51^{\text {st }}$ cycle is shown in Fig. 11(a) and a root square error (RSE) plot for comparing the

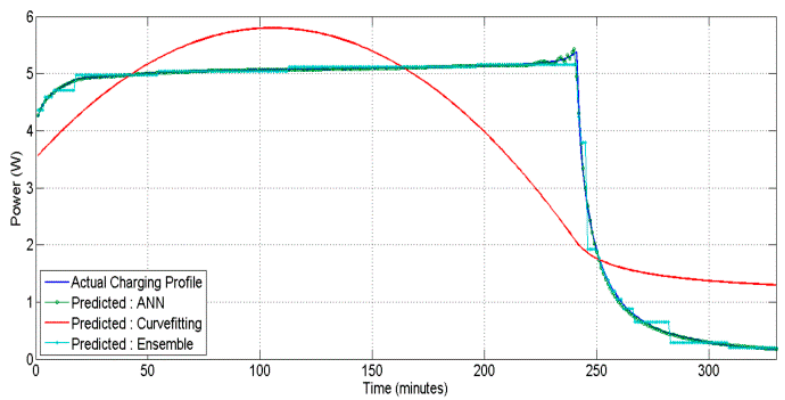

Fig. 10 (a). Predicted charging profile for $51^{\text {st }}$ cycle

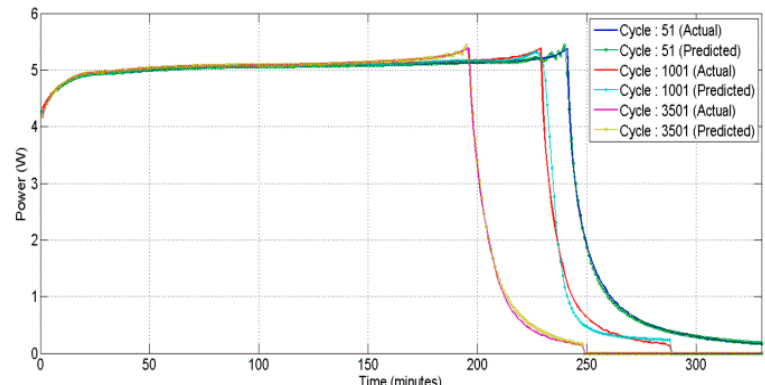

Fig. 10 (b). Predicted charging profile of the battery for 51 st cycle, 1001 st cycle, 3501 st cycle using ANN based model

accuracy of the ANN at different cycle is presented in Fig. 11(b). It can be observed from Fig. 11(a) that overall RSE of ANN based model is lower than other two methods. It can be observed from Fig. 11(b) that the RSE in some cases of CV region is higher irrespective the cycle number, otherwise the RSE is relatively lower. However, the mean absolute percentage error (MAPE) is $1.33 \%, 4.77 \%$ and $2.67 \%$ respectively for $51^{\text {st }}$ cycle, $1001^{\text {st }}$ cycle and $3501^{\text {st }}$ cycle. Similar to the case of simulated profiles, the error is significantly higher in $\mathrm{CV}$ region than in $\mathrm{CC}$ region.
The impact of number of neurons in the hidden layer on the errors is shown in Fig.12. It can be observed that when the number of neurons in hidden layer increases from ' 5 ' to ' 20 ', the errors decreases with the increase in number of neurons. However, when the number of neurons in hidden layer is more than 20 neurons, no significant change could be observed and it is evident from the case where 50 neurons are used in the hidden layer.

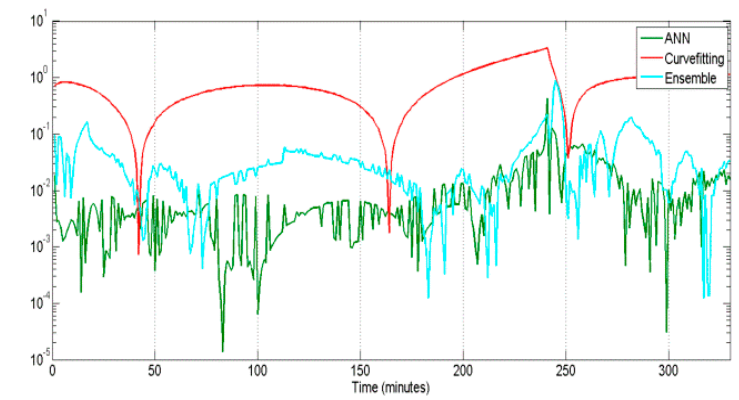

Fig. 11 (a). RSE plot for comparing the accuracy of the three models at $51^{\text {st }}$ cycle

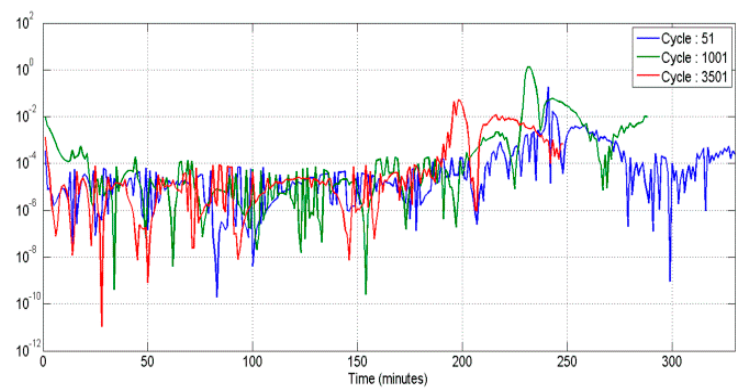

Fig. 11 (b). RSE plot for comparing the accuracy of the ANN at different cycle ANN based model
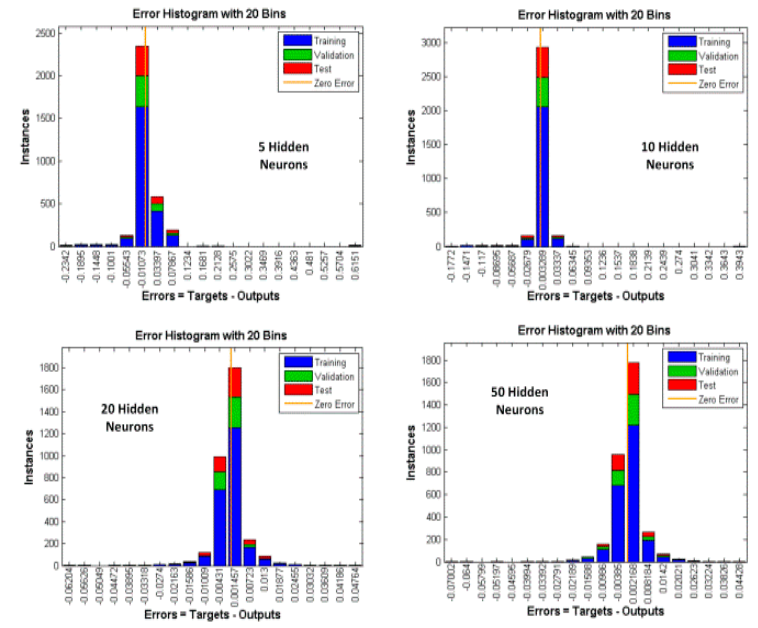

Fig. 12. Charging/discharging profile of the ESS on $131^{\text {st }}$ day of the year with ' $\varnothing$ ' $=2.5$ 


\section{CONCLUSION}

In this paper, application of different types of data-driven models namely ANN, Quadratic Polynomial curve fitting and Ensemble learning to predict the current charging profile of ESS based on previous charging profiles was examined. A methodology for utilizing such models was also proposed. The proposed method can leverage on the existing data from smart grid, hence does not need any additional investment or infrastructure. Furthermore, the data available could be used effectively and increases the value of the investment in smart grid infrastructure. The proposed method was applied to two different types of applications namely, applications where fixed power charging is employed and applications where variable power charging is employed. The models were also applied to predict the charging profiles of an $1100 \mathrm{mAh}, 3.6 \mathrm{~V}$ lithium iron phosphate battery, for which the charging profiles were collected over complete life cycle $(\sim 3500$ cycles). It was observed that the data-driven models especially ANN was able to predict the charging profile of ESS accurately, irrespective of the variations in different parameters. The proposed method could be deployed for various load management functions. The proposed approach needs minimal input such as initial SOC (only for mobile ESS) and smart meter data that would be available without any additional resources/infrastructure.

\section{ACKNOWLEDGMENT}

This work was supported by the Republic of Singapore's National Research Foundation through a grant to the Berkeley Education Alliance for Research in Singapore (BEARS) for the Singapore-Berkeley Building Efficiency and Sustainability in the Tropics (SinBerBEST) Program and the School of Electrical and Electronics Engineering, Nanyang Technological University. BEARS has been established by the University of California at Berkeley as a centre for intellectual excellence in research and education in Singapore.

\section{REFERENCES}

[1] A. H. Lim. HDB's Experience in Solar PV System [Online]. Available http://www.spring.gov.sg/NewsEvents/Events/Documents/SS601/7HDBExperienceinSolarPVSystem-AhHee.pdf

[2] R. Wong, "Solar Potential of HDB Blocks in Singapore," Energy Studies Institute Bulletin on Energy Trends and Development, vol. 4, pp. 6-7, 2011.

[3] 900 HDB blocks, eight govt sites to be equipped with solar panels [Online]. Available: https://www.edb.gov.sg/content/edb/en/news-andevents/news/2015-news/hdb-launches-first-tender-under-edb-ledprogramme-solarnova.html

[4] J. Wu, A. Botterud, A. Mills, Z. Zhou, B.-M. Hodge, and M. Heaney, "Integrating solar PV (photovoltaics) in utility system operations: Analytical framework and Arizona case study," Energy, vol. 85, pp. 1$9,2015$.

[5] N. Jenkins, Distributed generation: The Institution of Engineering and Technology, 2010.

[6] S. C. Vegunta, P. Twomey, and D. Randles, "Impact of PV and load penetration on LV network voltages and unbalance and potential solutions," 2013.

[7] T. Adefarati and R. Bansal, "Integration of renewable distributed generators into the distribution system: a review," IET Renewable Power Generation, vol. 10, pp. 873-884, 2016.
[8] P. Vithayasrichareon and I. F. MacGill, "Valuing large-scale solar photovoltaics in future electricity generation portfolios and its implications for energy and climate policies," IET Renewable Power Generation, vol. 10, pp. 79-87, 2016.

[9] D. Xu, H. Li, Y. Zhu, K. Shi, and C. Hu, "High-surety Microgrid: Super Uninterruptable Power Supply with Multiple Renewable Energy Sources," Electric Power Components and Systems, vol. 43, pp. 839$853,2015$.

[10] L. Barelli, U. Desideri, and A. Ottaviano, "Challenges in load balance due to renewable energy sources penetration: The possible role of energy storage technologies relative to the Italian case," Energy, vol. 93, pp. 393-405, 2015.

[11] P. Denholm, E. Ela, B. Kirby, and M. Milligan, "The role of energy storage with renewable electricity generation," 2010.

[12] S. A. Hamidi, D. M. Ionel, and A. Nasiri, "Modeling and Management of Batteries and Ultracapacitors for Renewable Energy Support in Electric Power Systems-An Overview," ELECTRIC POWER COMPONENTS AND SYSTEMS, vol. 43, pp. 1434-1452, 2015.

[13] D. Fooladivanda, C. Rosenberg, and S. Garg, "Energy Storage and Regulation: An Analysis," IEEE Transactions on Smart Grid, vol. 7, pp. 1813-1823, 2016.

[14] D. Panahi, S. Deilami, M. A. Masoum, and S. M. Islam, "Forecasting plug-in electric vehicles load profile using artificial neural networks," in Power Engineering Conference (AUPEC), 2015 Australasian Universities, 2015, pp. 1-6.

[15] K. Nandha, P. Cheah, B. Sivaneasan, P. So, and D. Wang, "Electric vehicle charging profile prediction for efficient energy management in buildings," in IPEC, 2012 Conference on Power \& Energy, 2012, pp. 480-485.

[16] R. Badrinarayanan, J. Zhao, K. Tseng, and M. Skyllas-Kazacos, "Extended dynamic model for ion diffusion in all-vanadium redox flow battery including the effects of temperature and bulk electrolyte transfer," Journal of Power Sources, vol. 270, pp. 576-586, 2014.

[17] D.-I. Stroe, V. Knap, M. Swierczynski, A. Stan, and R. Teodorescu, "Operation of Grid-Connected Lithium-Ion Battery Energy Storage System for Primary Frequency Regulation: A Battery Lifetime Perspective," IEEE Transactions on Industry Applications, 2016.

[18] J. Wang, P. Liu, J. Hicks-Garner, E. Sherman, S. Soukiazian, M. Verbrugge, et al., "Cycle-life model for graphite-LiFePO 4 cells," Journal of Power Sources, vol. 196, pp. 3942-3948, 2011.

[19] L. Lam and P. Bauer, "Practical capacity fading model for Li-ion battery cells in electric vehicles," IEEE transactions on power electronics, vol. 28, pp. 5910-5918, 2013

[20] P. Ramadass, B. Haran, R. White, and B. N. Popov, "Mathematical modeling of the capacity fade of Li-ion cells," Journal of Power Sources, vol. 123, pp. 230-240, 2003

[21] N. K. Kandasamy, R. Badrinarayanan, V. R. K. Kanamarlapudi, K. J. Tseng, and B.-H. Soong, "Performance Analysis of Machine-Learning Approaches for Modeling the Charging/Discharging Profiles of Stationary Battery Systems with Non-Uniform Cell Aging," Batteries, vol. 3, p. 18, 2017.

[22] Singapore land transport statistics (2014) in brief [Online]. Available: https://www.lta.gov.sg/content/dam/ltaweb/corp/PublicationsResearch/ files/FactsandFigures/StatisticsinBrief2014.pdf

[23] A. Ashtari, E. Bibeau, S. Shahidinejad, and T. Molinski, "PEV charging profile prediction and analysis based on vehicle usage data," IEEE Transactions on Smart Grid, vol. 3, pp. 341-350, 2012.

[24] D. F. R. Melo, G. H. Beng, and T. Massier, "Charging of electric vehicles and demand response management in a Singaporean car park," in Power Engineering Conference (UPEC), 2014 49th International Universities, 2014, pp. 1-6.

[25] S. Sarkar, D. K. Jha, A. Ray, and Y. Li, "Dynamic data-driven symbolic causal modeling for battery performance \& health monitoring," in Information Fusion (Fusion), 2015 18th International Conference on, 2015, pp. 1395-1402.

[26] Y. Li, P. Chattopadhyay, S. Xiong, A. Ray, and C. D. Rahn, "Dynamic data-driven and model-based recursive analysis for estimation of battery state-of-charge," Applied Energy, vol. 184, pp. 266-275, 2016.

[27] Z.-S. Hou and Z. Wang, "From model-based control to data-driven control: survey, classification and perspective," Information Sciences, vol. 235, pp. 3-35, 2013. 